ARTICLE

https://doi.org/10.1038/s41467-019-13948-y

\title{
Targeting the tumor vasculature with engineered cystine-knot miniproteins
}

Bonny Gaby Lui ${ }^{1}$, Nadja Salomon ${ }^{1}$, Joycelyn Wüstehube-Lausch ${ }^{1}$, Matin Daneschdar ${ }^{1}$, Hans-Ulrich Schmoldt ${ }^{1}$, Özlem Türeci $\&$ Ugur Sahin ${ }^{1 \star}$

The extra domain B splice variant (EDB) of human fibronectin selectively expressed in the tumor vasculature is an attractive target for cancer imaging and therapy. Here, we describe the generation and characterization of EDB-specific optical imaging probes. By screening combinatorial cystine-knot miniprotein libraries with phage display technology we discover exquisitely EDB-specific ligands that share a distinctive motif. Probes with a binding constant in the picomolar range are generated by chemical oligomerization of selected ligands and fluorophore conjugation. We show by fluorescence imaging that the probes stain EDB in tissue sections derived from human U-87 MG glioblastoma xenografts in mice. Moreover, we demonstrate selective accumulation and retention of intravenously administered probes in the tumor tissue of mice with U-87 MG glioblastoma xenografts by in vivo and ex vivo fluorescence imaging. These data warrants further pursuit of the selected cystine-knot miniproteins for in vivo imaging applications. 
C omputed tomography and magnetic resonance imaging are routinely used in cancer diagnosis and for monitoring of treatment outcome ${ }^{1}$. Targeted molecular imaging is emerging as an attractive complementary technology, as it is capable of visualizing tumors with high precision, specificity, and resolution by using molecular reagents directed against cancerassociated proteins ${ }^{2}$. Cell surface molecules associated with tumor angiogenesis that are expressed at high levels in the tumor blood vessels and hence are easily accessible from the bloodstream are considered as attractive targets ${ }^{3}$.

Fibronectin is a broadly expressed component of the extracellular matrix. Its splicing isoform, containing the extra domain $\mathrm{B}$ (EDB), is overexpressed during embryonic development and in a variety of solid tumors including brain cancer, whereas it is virtually undetectable in normal adult tissues ${ }^{4,5}$. EDB is a type III domain of 91 amino acids inserted between fibronectin (FN) domains 7 and 8 of the oncofetal fibronectin isoform ${ }^{6,7}$. The EDB sequence is highly conserved across species and the orthologous protein sequences in mice, rats, rabbits, and humans are completely identical ${ }^{5}$.

Being a selective tumor marker, EDB has been explored as a target for cancer imaging and therapy in early clinical trials ${ }^{8}$. L19, the most advanced EDB-selective ligand thus far, was isolated from an antibody phage display library by Neri and colleagues 9 . The L19 antibody has been investigated for various diagnostic and therapeutic applications ${ }^{10-13}$. As the large molecular size and complex folding of antibodies pose manufacturing challenges ${ }^{14}$, development of smaller EDB-specific ligands is being explored. These include ligands with nanomolar-binding affinities based on Fynomers, Anticalins, and an ubiquitin-derived protein selected from combinatorial phage libraries ${ }^{15,16,17}$. Moreover, two EDBrecognizing peptides have been generated and conjugated with fluorescent dyes for preclinical in vivo imaging studies. One study reports effective visualization of human prostate tumor xenografts with a cyclic nonapeptide called ZD2 $2^{18}$. In another study, artificial high-affinity peptides (so-called aptides) have been shown to specifically target human glioblastoma tumor xenografts $^{19}$. Altogether, these data have further boosted the interest in the concept of EDB targeting probes ${ }^{8}$.

In recent years, the cystine-knot miniprotein or knottin family with its superior pharmacological properties has gained particular attention for in vivo imaging and targeted drug delivery ${ }^{20-22}$. Cystine-knot miniproteins are small (30-50 amino acids in size) disulfide-rich peptides with extraordinarily high thermal, proteolytic, and chemical stability. Their simple architecture allows convenient chemical manufacturing and site-directed conjugation of, e.g. fluorophores, chelators, or toxins ${ }^{23,24}$. The exposed surface loops of cystine-knot miniproteins tolerate sequence modifications and insertions, facilitating the generation of target-binding capabilities via loop grafting or directed evolution based protein design. Cystine-knot miniprotein family members, including Momordica cochinchinensis trypsin inhibitor II (MCoTI-II), Spinacia oleracea trypsin inhibitor (SOTI), and Ecballium elaterium trypsin inhibitor II (EETI), have been engineered as specific binders against a variety of target proteins ${ }^{25}$.

This study describes the characterization of EDB-binding cystine-knot miniproteins, which are discovered by screening of a combinatorial phage display library based on an open chain variant of the trypsin inhibitor II from Momordica cochinchinensis (oMCoTI-II). MC-FN-010 and its derivative MC-FN-016 are selected for oligomerization and fluorescent dye conjugation to obtain trimeric imaging probes. These probes show specific in vivo tumor targeting properties in a glioblastoma xenograft mouse model, while they have low overall background signals. Our findings demonstrate the high potential of cystine-knot miniproteins for development of molecular imaging agents.

\section{Results}

Discovery of EDB-specific cystine-knot miniproteins. For the selection of EDB-specific cystine-knot miniproteins, two different M13 phage libraries based on the open chain sequence of oMCoTI-II $^{26}$ were used. The MCopt 1.0 library comprises sequences with randomized amino acids in the first loop, scattered positions in the third loop, and two variable residues upstream of the first cysteine, and is presented via the pVIII major coat protein, resulting in a polyvalent type of display. The MCopt 2.0 library, in contrast, is displayed via the minor coat protein (pIII) and contains a randomized stretch of 10 amino acids in the first loop only (Fig. 1a).

For the screening and the hit identification process we used a protein fragment representing the single EDB domain (FN-B). Hexahistidine (H6)-tagged FN-B was recombinantly expressed in E. coli and purified via immobilized metal ion affinity chromatography (IMAC) and size exclusion chromatography (SEC) to a purity of $>90 \%$ (Supplementary Fig. 1a). In addition, EDB flanked by its surrounding type III domains (FN-67B89) and an analogous variant without inserted EDB (FN-6789), mimicking the respective epitope in healthy tissues, were generated as control proteins for downstream assays. Identity was confirmed by detecting the C-terminal H6-tag (Supplementary Fig. 1b). Native folding of FN-67B89 was verified in a enzyme-linked immunosorbent assay (ELISA)-based assay with a monoclonal antibody (BC-1), which distinguishes between fibronectin containing $\mathrm{EDB}$ and fibronectin without $\mathrm{EDB}^{27}$ (Supplementary Fig. 1b).

Both phage libraries were screened in three consecutive rounds against biotinylated FN-B and 46 single clones were selected for sequencing. Screening of the MCopt 1.0 library resulted in strong enrichment of one single cystine-knot miniprotein (MC-FN-030) comprising $40 \%$ of all sequences in the pool. Two other frequent clones were identified, representing $4 \%$ and $2 \%$ (MC-FN-020, MC-FN-010), respectively, of the repertoire (Fig. 1b). The sequences harvested from the third screening round were fused N-terminally to Thioredoxin-A and to a H6- and S-tag by subcloning and the respective Trx-cystine-knot miniproteins were expressed in a 96-well mini scale format (Fig. 1a). Binding of proteins in this pool to FN-B was tested in an ELISA-based assay and the expression rate of each clone was determined via 96-well SDS-PAGE (E-PAGE ${ }^{\mathrm{TM}}$ ) analysis. Based on the derived signal-tonoise ratio and the expression value, we calculated a ranking score for each Trx-cystine-knot miniprotein as a measure for FN-B interaction. Three candidates showed higher interaction with FN$B$ than with bovine serum albumin (BSA) as control. These sequences with confirmed FN-B binding were found to correspond to the enriched clones (Supplementary Table 1).

Screening of the second library, MCopt 2.0, resulted in enrichment of three different cystine-knot miniproteins to $13 \%$, $10 \%$, and 2\% (MC-FN-050, MC-FN-060, MC-FN-040), respectively, of all sequences. Sequence analysis of all six clones derived from the two library screens revealed that five of these sequences (all except MC-FN-020) shared a common R-I/V-R-(L) motif at the C-terminal end of loop 1 (Fig. 1b). All six clones (those derived from MCopt 2.0 without further confirmation via binding ELISA) were subjected to further analysis.

Binding specificity of the six candidates obtained by screening of the two libraries was analyzed by testing against various protein fragments containing the EDB domain alone or inserted in its natural or an artificial protein sequence, e.g. FN-B (with which the screening had been performed), FN-67B89, T7-TEV-B (a T7-His-TEV tag N-terminally flanked EDB domain), and FN$\mathrm{B}(8-14)$ (EDB protein with its C-terminal domains 8-13 and a part of domain 14) (Fig. 2a) (for quality controls see Supplementary Fig. 1a). FN-6789 was used as off-target control, 
a

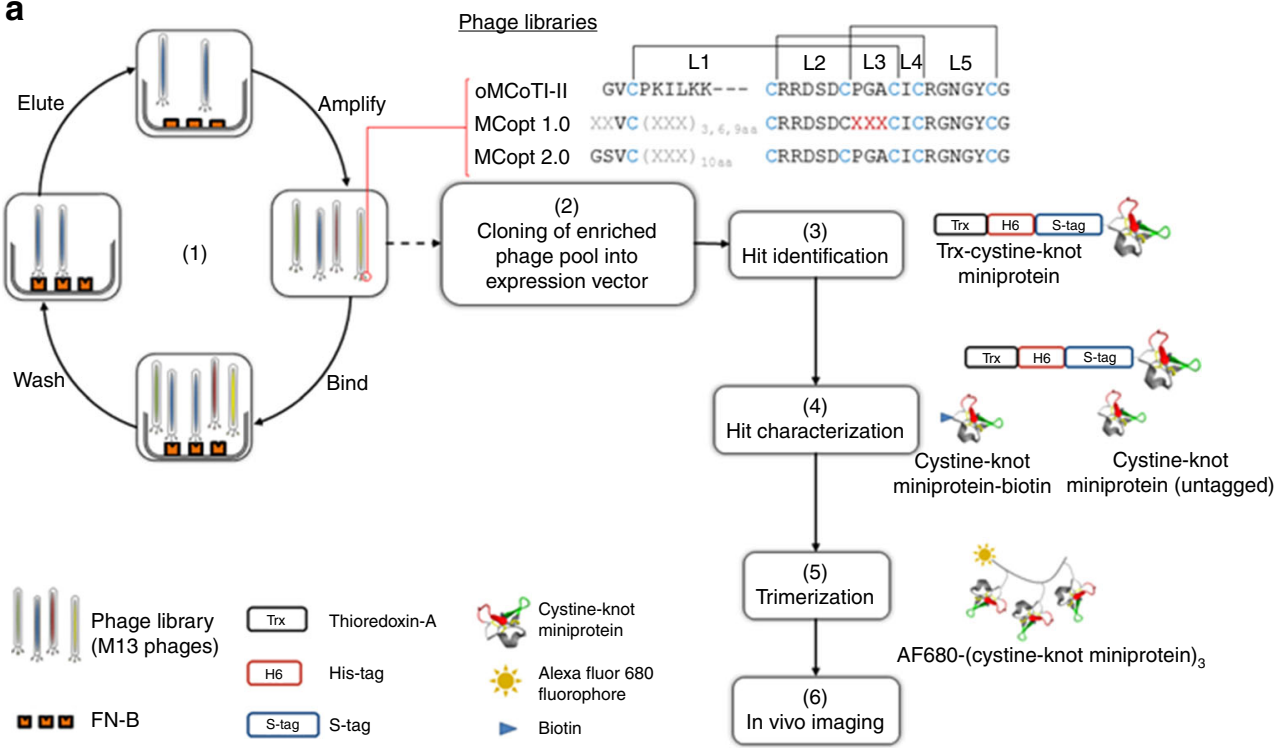

b

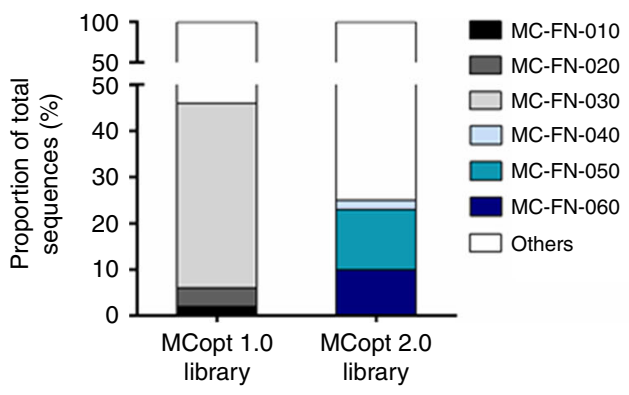

Enriched cystine-knot miniprotein sequence

PMC-TQRKNRIRLCRRDSDCTGACICRGNGYCG WTC-TKKYPNTI SCRRDSDCRVTCICRGNGYCG WKC-QPTNGYRI RCRRDSDCPGDCICRGNGYCG SVCAHYNT IRVRLCRRDSDCPGACICRGNGYCG SVCKNVS IMRI RLCRRDSDCPGAC ICRGNGYCG SVCKQANFVRIRLCRRDSDCPGACICRGNGYCG

Fig. 1 Enrichment of clones with a common sequence motif by library screening against EDB. a EDB-specific ligand selection and development of an imaging agent. (1) Three successive rounds of screening of MCopt 1.0 and MCopt 2.0 phage libraries (both based on the oMCoTI-II sequence framework) were performed against a hexahistidine (H6)-tagged single EDB-domain (FN-B) fragment. Disulfide bonds (brackets) between cysteine residues (blue), randomized positions for any random amino acid except cysteine ( $X$ in gray), and amino acid substitutions to $50 \%$ ( $X$ in red) are indicated. L1 to L5 represent the loop positions. (2) Cystine-knot miniprotein sequences were cloned into expression vector for Trx-cystine-knot miniprotein production. (3) Hit identification of individual clones was performed by ELISA-based binding analysis (Trx-cystine-knot miniprotein), determination of expression rate, and sequencing. (4) Hits were characterized with regard to affinity (with untagged cystine-knot miniprotein), specificity (Trx-cystine-knot miniprotein, cystineknot miniprotein-biotin), and functionality (Trx-cystine-knot miniprotein). (5) Trimerization of lead cystine-knot miniprotein candidates and Alexa Fluor 680 fluorophore conjugation was performed to allow (6) imaging of tumor vasculature in vivo in a mouse model xenografted with a human glioblastoma cell line. $\mathbf{b}$ Enrichment of cystine-knot miniprotein sequences after three screening rounds of phage display libraries MCopt 1.0 and MCopt 2.0. Variable amino acids (blue letters) and the common R-I/V-R-(L) motif (red) are indicated.

as fibronectin lacking EDB is expressed by many different cell types $^{28}$, whereas the EDB variant is selective for tumor vasculature.

All clones were found to bind strongly to all recombinant fragments containing EDB (Fig. 2b, Supplementary Fig. 2). The five variants containing the common R-I/V-R-(L) motif showed low or moderate binding to off-target and control proteins, with MC-FN-060 having the highest background signal. For MC-FN020 , which does not contain the R-I/V-R-(L) motif, there was strong binding to the off-target control FN-6789 and the other control proteins (streptavidin and milk powder) except BSA. These data strongly indicate that the identified amino acid motif is relevant for selectivity of EDB binding.

Dose titration of the five R-I/V-R-(L)-motif-containing candidates revealed apparent $\mathrm{KD}$ values for binding to $\mathrm{FN}-\mathrm{B}$ and FN-67B89, respectively, in the nanomolar range (6-235 nM) (Fig. 2c), with MC-FN-010 being the strongest binder. Binding to control FN-6789 was measurable at concentrations higher than one order of magnitude. Again, clone MC-FN-060 showed the highest non-specific binding to the FN-6789 off-target control.

These experiments had been performed with the tagged versions (Thioredoxin-A-, H6-, and S-tag) of the respective cystine-knot miniproteins. As for the purpose of being used as in vivo imaging tool any tags have to be removed, we next studied the binding ability of tag-free variants of the four strongest binders by surface plasmon resonance (SPR) analysis. Surprisingly, only MC-FN-010 showed detectable binding activity towards the FN-67B89 target protein at concentrations ranging from 50 to $1000 \mathrm{nM}$ (Fig. 2d). Therefore, we focused on MC-FN010 for further studies.

Systematic analysis of the R-I/V-R-(L)-binding motif. The occurrence of the amino acid motif R-I/V-R-(L) in the enriched and specifically binding cystine-knot miniproteins suggested involvement of this sequence in EDB binding. We performed a systematic alanine scanning mutagenesis of MC-FN-010, in 
a Full-length FN with its splice domains

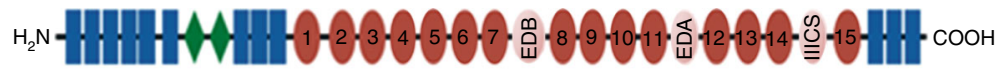

\begin{tabular}{|c|c|c|}
\hline FN-B & $\mathrm{H}_{2} \mathrm{~N}=\stackrel{\vec{山}}{\mathrm{u}}=\mathrm{COOH}$ & Type I domain \\
\hline FN-67B89 & & Type II domain \\
\hline $\mathrm{FN}-\mathrm{B}(8-14)$ & & Type III domain \\
\hline T7-His-TEV-B & ·물- & $\begin{array}{l}\text { Type III, } \\
\text { splice-in domain }\end{array}$ \\
\hline FN-6789 & & 29aa T7-His-TE \\
\hline
\end{tabular}
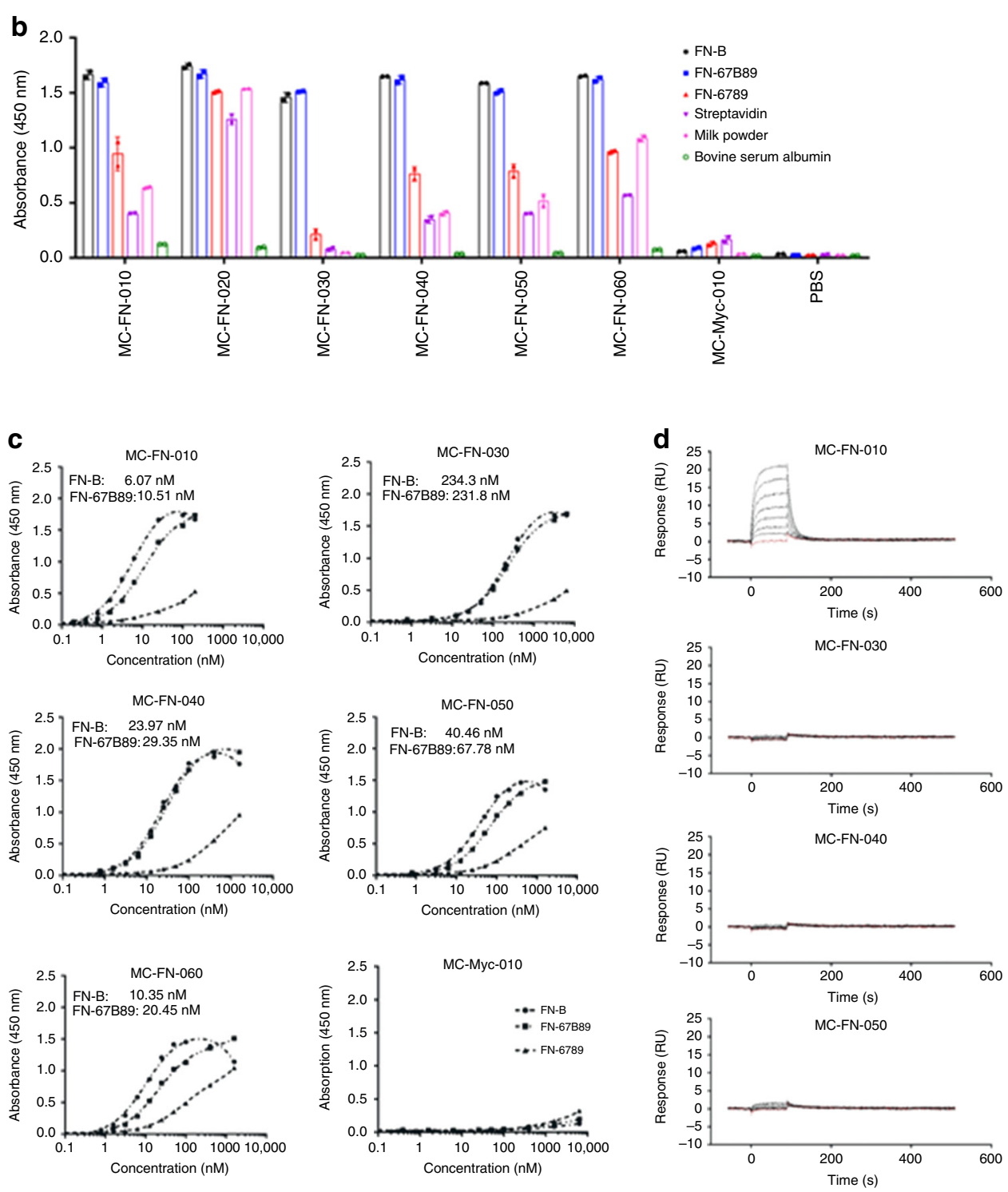

which every single amino acid in the variable region and the arginine residues in loops 2 and 5 (L2 and L5, see Fig. 1a) were individually exchanged for alanine. All constructs were tested for binding to single domain FN-B (Fig. 3a). Four constructs with exchanges of single amino acids within the common motif positions had lower binding as compared to that of the respective original sequence. An additional alanine substitution in the fifth loop also led to reduced target interaction. In contrast, target binding was preserved in the seven constructs with alanine exchanges in the beginning of the sequence, indicating that those positions were not crucial for EDB binding. Together, these results confirm that the four amino acid residues in the first loop 
Fig. 2 Discovery of cystine-knot miniproteins with specific binding to EDB. a EDB-containing protein fragments used in this study. The EDB domain distinguishes the oncofetal variant of fibronectin from the ubiquitously expressed fibronectin. FN-B (single domain), FN-67B89 (EDB flanked by the naturally surrounding type III domains), FN-B(8-14) (EDB protein with its C-terminal domains 8-13 and a part of domain 14), T7-His-TEV-B (N-terminally tagged EDB domain), and FN-6789 (the type III domains 6-9 without EDB). b ELISA-based specificity analysis of enriched cystine-knot miniproteins. In all, $200 \mathrm{nM}$ of each Trx-cystine-knot miniproteins were applied to immobilized FN-B and FN-67B89 target proteins and to control proteins FN-6789, streptavidin, milk powder, and BSA (coated at $1 \mu \mathrm{g} /$ well). Data are shown as mean \pm SD. c EDB-binding curves of Trx-cystine-knot miniproteins were generated against FN-B and FN-67B89 targets and against FN-6789 control protein (coated at $1 \mu \mathrm{g} /$ well). Binding was detected with an HRP-conjugated anti-S-tag antibody. ELISA was performed in duplicates in single measurement and with MC-Myc-010, harboring a Myc epitope in loop 1, serving as a negative control. The apparent KD-value was calculated based on non-linear regression fitting curves. $\mathbf{d}$ SPR sensorgrams of a multi-cycle kinetic binding analysis of selected cystine-knot miniproteins. Biotinylated FN-67B89 was captured on a streptavidin sensor chip and untagged cystine-knot miniproteins were analyzed with concentrations ranging from 50 to $1000 \mathrm{nM}$ (curves from bottom down to top). The curves were referenced to a blank streptavidin control flow cell. Source data are provided as a Source Data file.
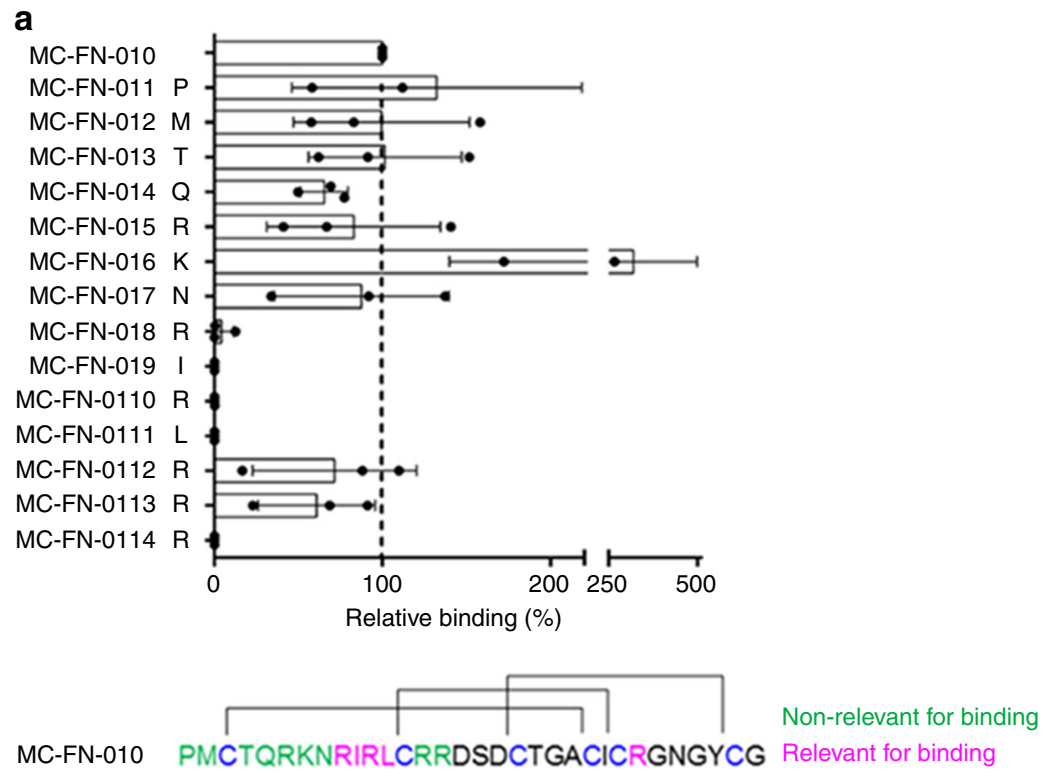

b

MC-FN-010

PMCTQRKNRIRLCRRDSDCTGACICRGNGYCG

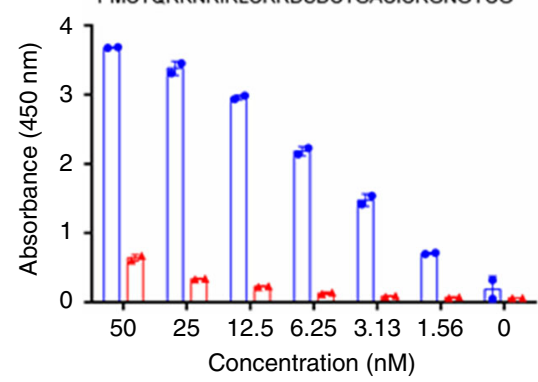

MC-FN-016

PMCTQRANRIRLCRRDSDCTGACICRGNGYCG

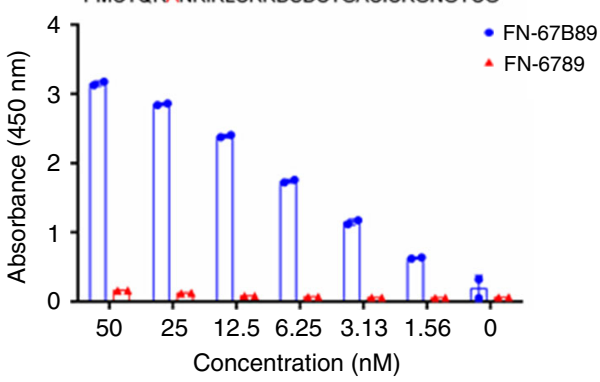

Fig. 3 Dependency of EDB binding on the R-I/V-R-(L) motif and further sequence optimization. a Identification of functionally relevant amino acids in the MC-FN-010 sequence by alanine scanning mutagenesis. Trx-cystine-knot miniproteins (parental MC-FN-010 and alanine scanning variants) were incubated with pre-coated human FN-B. Binding was detected with HRP-conjugated anti-S-tag antibody. Relative binding of each variant was calculated by comparing the respective apparent binding constant to parental MC-FN-010. Error bars represent mean \pm SD of three independent duplicate measurements. EDB binding relevant amino acid residues of parental MC-FN-010. Disulfide bonds (brackets) between cysteine residues (blue), residues relevant (magenta), or at best marginally relevant (green) for target binding are indicated. $\mathbf{b}$ ELISA-based binding analysis of MC-FN-010 and MC-FN-016 to human FN-67B89 and FN-6789 (1 $\mu \mathrm{g} /$ well). Trx-cystine-knot miniproteins were applied at different concentrations in duplicates and binding was detected with HRP-conjugated anti-S-tag antibody. Data shown as mean \pm SD. Source data are provided as a Source Data file.

(RIRL), and the arginine residue in the fifth loop impact binding to EDB either directly or indirectly by affecting miniprotein conformation.

This analysis also identified mutagenized variants, which were comparable with regard to binding to the EDB domain. We leveraged these data for selection of a second development candidate, with which we could also address that the lysine residue in loop 1 of MC-FN-010 may interfere with the selective linkage to primary amines required for full exploitation as an imaging reagent. MC-FN-016 had a primary amine only at the 
Table 1 SPR-binding analysis of cystine-knot miniprotein variants to EDB.

\begin{tabular}{|c|c|c|c|c|}
\hline MC-FN-016 & $62.5-4000$ & $1.33 \times 10^{5}$ & 0.1873 & 1410 \\
\hline AF680-(MC-FN-016) 3 & $1.25-10$ & $2.56 \times 10^{6}$ & $2.31 \times 10^{-3}$ & 0.902 \\
\hline$(\mathrm{MC}-\mathrm{FN}-010)_{3}$ & $1.25-10$ & $4.82 \times 10^{4}$ & $1.84 \times 10^{-4}$ & 3.82 \\
\hline AF680-(MC-FN-0115) 3 & $1.25-10$ & 0 & 0 & 0 \\
\hline
\end{tabular}

$\mathrm{N}$-terminus, and was found to be comparable to MC-FN-010 with regard to its specificity for binding to FN-67B89 (Fig. 3b). The affinities of tag-free MC-FN-010 and MC-FN-016 towards biotinylated FN-67B89 as tested by SPR analysis were in the single-digit micromolar range with fast off-rates (Table 1, Supplementary Fig. 3a).

Tumor vasculature targeting with cystine-knot miniproteins. Glioblastomas are known to express the fibronectin EDB isoform in their vasculature 29 and cell line models derived from this tumor type are commonly used for in vitro and in vivo characterization of EDB-binding ligands ${ }^{30,31}$. We resorted to a mouse model based on inoculating the human glioblastoma cell line U87 MG subcutaneously into the flanks of Fox n1/nu mice to obtain xenograft tumors for ex vivo analysis and in vivo studies.

First, we tested MC-FN-010 staining on tissue sections derived from these xenograft tumors. MC-FN-0115 was used as nonbinding negative control (alanine substitutions in three positions; PMCTQRANRIAACRRDSDCTGACICRGNGYCG) (Supplementary Fig. 4) and biotinylated MC-FN-010 and MC-FN-0115 were tetramerized with Cy3-labeled streptavidin to increase avidity. On U-87 MG tumor sections tetramerized MC-FN-010bio bound almost selectively to areas around tumor vessels and co-localized with the vascular marker CD31, a surface protein ubiquitously expressed on endothelial cells, whereas MC-FN0115-bio did not stain these sections at all. In addition, tetramerized MC-FN-010-bio was found to localize in perivascular areas. Normal mouse brain sections were not stained by MC-FN-010-bio (Fig. 4a), indicating specificity for tumor vasculature.

Next, for assessing MC-FN-010 and MC-FN-016 in vivo, the cystine-knot miniproteins were chemically trimerized by oxime ligation and tagged with a near-infrared fluorescent dye, Alexa Fluor 680 (Fig. 1a). Oligomerization profoundly improved affinity of both EDB-binding miniproteins. The trimers ((MC-FN-010) AF680-(MC-FN-010) $)_{3}$, and AF680-(MC-FN-016) ${ }_{3}$ ) exhibited binding constants in the three-digit picomolar range and had remarkably slower off-rates as compared to the monomeric variants (Table 1, Supplementary Fig. 3b). EDB-binding properties of the cystine-knot miniproteins were not altered by trimerization as confirmed by immunofluorescence staining of tumor and normal brain tissue sections (Supplementary Fig. 5).

U-87 MG xenografted mice were injected intravenously with $3.34 \mathrm{nmol}$ AF680-(MC-FN-010) $)_{3}$, AF680-(MC-FN-016) 3 , or the negative control AF680-(MC-FN-0115) $)_{3}$, and whole-body imaging and ex vivo measurement of resected organs was performed. In a parallel experiment, mice were injected with 10 -fold excess of unlabeled (MC-FN-010) 3 as competitor along with $3.34 \mathrm{nmol}$ AF680-(MC-FN-010) 3 to compare the kinetics of tumor fluorescence intensity to the in vivo experiment without competition. Both AF680-(MC-FN-010) ${ }_{3}$ and AF680-(MC-FN-016) 3 but not the control variant strongly accumulated in the tumor within the first hours and remained significantly enriched over the recorded $6 \mathrm{~h}$ time period (Fig. 4b, c). As a consequence, analysis of resected tissues showed that tumor-to-organ ratios of AF680-(MC-FN$010)_{3}$ and AF680-(MC-FN-016) 3 for organs, which are of relevance as frequent sites of metastasis in clinical imaging, increased over time and enabled improved capture of the specific signal (Fig. 4d, Table 2). As demonstrated by ex vivo imaging, the tumor signals in mice treated with the competitor were substantially reduced at each time point (Supplementary Fig. 6). The resulting tumor-to-organ ratios significantly increased over time demonstrating a strong EDB specificity of AF680-(MC-FN$010)_{3}$ (Fig. 4e).

We compared the in vivo tumor targeting of AF680-(MC-FN$010)_{3}$ to two previously described EDB-binding peptides (aptide ${ }^{17}$ and nonapeptide $\left.{ }^{19}\right)$. In the comparative study, 3.34 nmol AF680(MC-FN-010) 3 , AF680-aptide, and AF680-nonapeptide were intravenously administered to U-87 MG bearing mice (Supplementary Fig. 7, Supplementary Table 2). In accordance to our previous observations, AF680-(MC-FN-010) 3 was strongly enriched in the tumors and tumor-to-organ ratios increased markedly over time. In contrast, AF680-aptide was predominantly detected in the liver and kidney with low tumor-to-organ ratios. For detectability of fluorescence signals of AF680nonapeptide in the tumor at $1 \mathrm{~h}$ post-injection, a higher dose $(10 \mathrm{nmol})$ of the probe had to be administered (Supplementary Fig. 8).

In aggregate, these data warrants to further explore the identified EDB-specific cystine-knot miniproteins for use as imaging agents for tumor targeting.

\section{Discussion}

Targeted tumor imaging with peptide- or protein-based molecular probes may improve cancer diagnosis, staging, and treatment monitoring ${ }^{32}$. The selective expression of the EDBcontaining isoform of Fibronectin in tumor-associated neovasculature $^{7}$ makes it an ideal target for ligand-based delivery of drug molecules. Derivatives of the EDB-binding human L19 antibody have been widely used as a molecular system to deliver radioisotopes or cytokines in cancer imaging and therapy ${ }^{33-35}$. Several L19-based approaches are currently under clinical investigation with promising early results ${ }^{36,37}$. This together with the fact that cystine-knot miniproteins entail a broad range of favorable characteristics as compared to a full antibody format encouraged us to generate EDB-binders based on this scaffold.

In this study we present the development of cystine-knot peptide-based ligands with high specificity for fibronectin EDB and its preclinical evaluation as an in vivo imaging probe in a glioblastoma mouse model. The screening of two combinatorial libraries against an EDB-containing protein fragment resulted in strong enrichment of six clones, five of which shared the distinctive R-I/V-R-(L) motif. We characterized the identified hits and selected MC-FN-010 based on the robust affinity and 
a

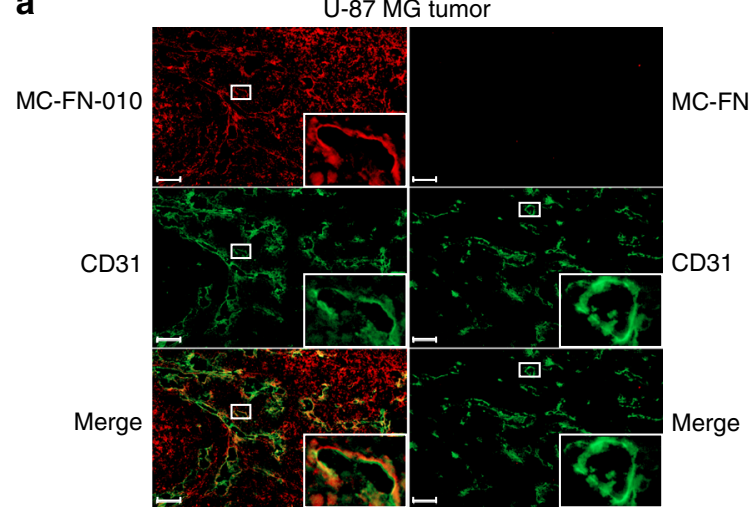

Brain

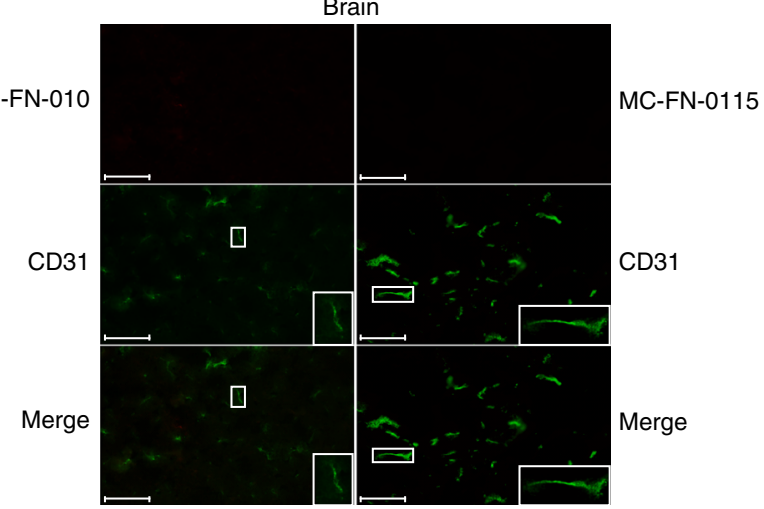

b

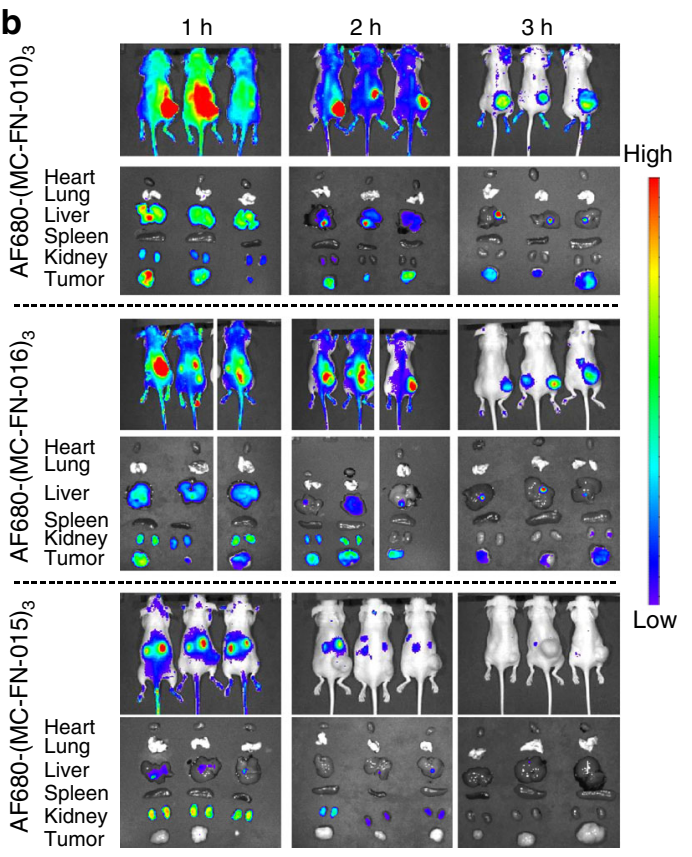

d
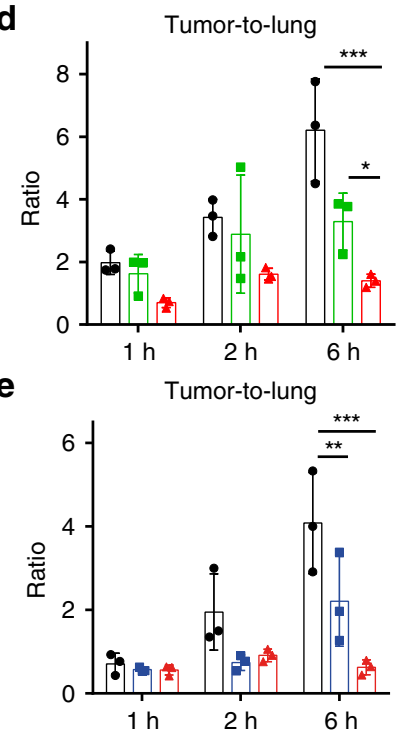
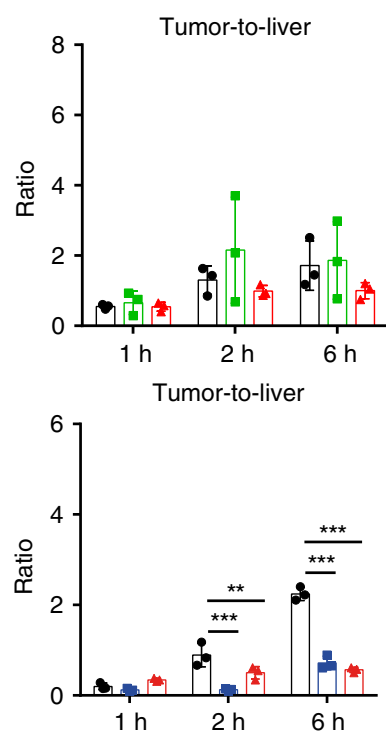
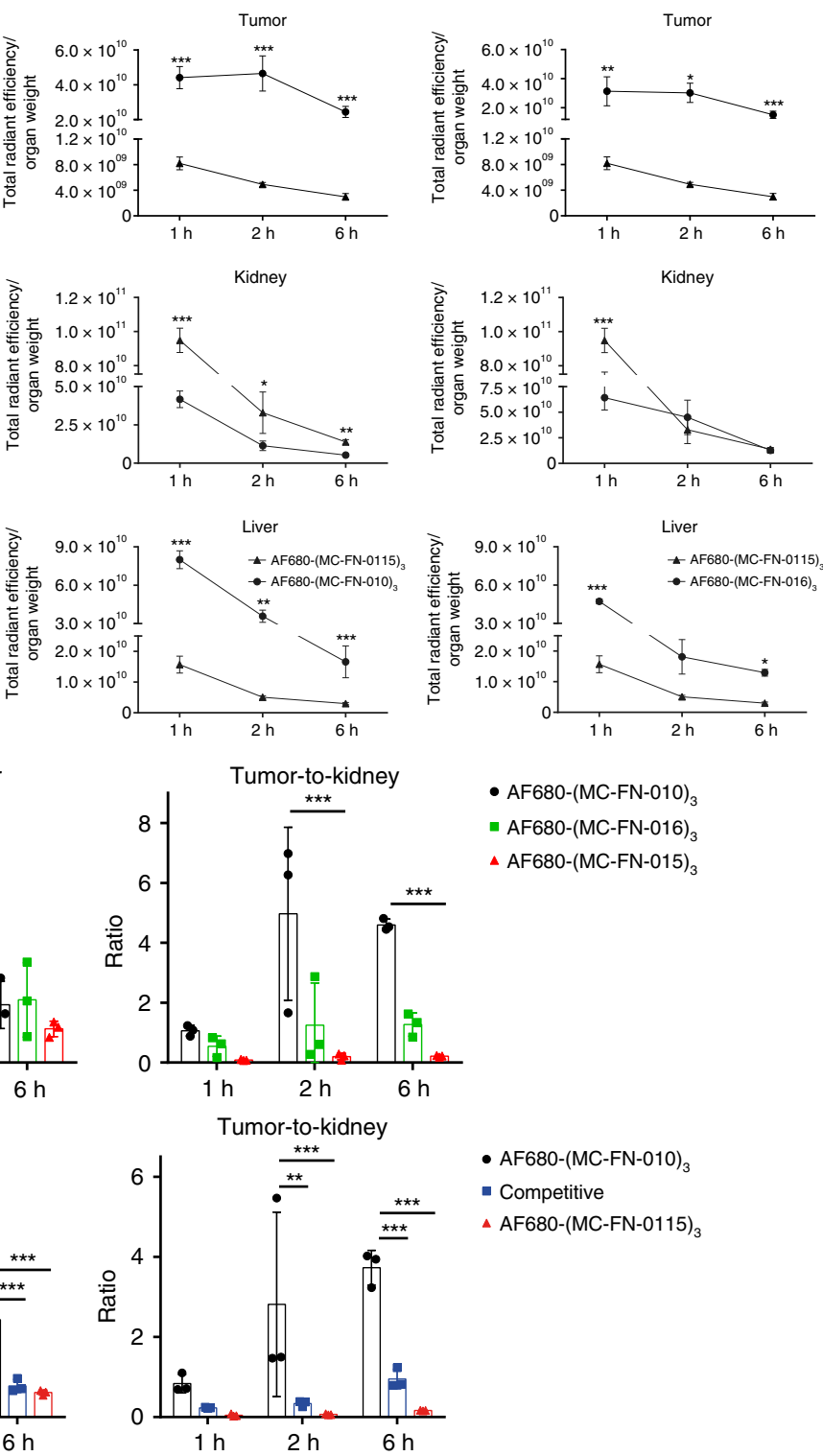

- $\mathrm{AF} 680-(\mathrm{MC}-\mathrm{FN}-010)_{3}$

- AF680-(MC-FN-016) $\triangle \mathrm{AF} 680-(\mathrm{MC}-\mathrm{FN}-015)_{3}$

specificity of its binding to EDB for further development. Mapping of the MC-FN-010-binding site through alanine scanning mutagenesis revealed that the amino acid residues $\mathrm{R}$ $\mathrm{I}-\mathrm{R}-\mathrm{L}$ in the first loop and an arginine residue in the fifth loop are required for EDB-binding activity of the peptide. One may speculate that the consensus sequence together with amino acids located in loop 2 (R-R-D-S-D) and loop 5 (R-G-N-G-Y) forms a patch of positively charged residues that mediate binding to EDB mainly via electrostatic interactions. This hypothesis is supported by the dominance of negative charges with only two positively charged residues being represented within the 91 amino acids of $\mathrm{EDB}^{38}$. 
Fig. 4 Targeting the tumor vasculature with selected cystine-knot miniproteins. a Specific binding of MC-FN-010 to tissue sections derived from the human U-87 MG glioblastoma cell line grown as mouse xenograft tumor. Representative immunofluorescence staining of U-87 MG tumor tissues and normal mouse brain with EDB ligand MC-FN-010 and negative control MC-FN-0115. Tissue sections ( $6 \mu \mathrm{m})$ were stained with tetramerized cystine-knot miniprotein-biotin/strepatividin-Cy3 complex (red) and an anti-CD31 antibody to visualize vasculature (green). Scale bars, $100 \mu \mathrm{m}$. $\mathbf{b} \mathrm{In}$ vivo and ex vivo imaging of U-87 MG bearing mice. Tumors derived from human U-87 MG cells injected s.c. in flanks of mice were imaged after i.v. application of 3.34 nmol AF680-(MC-FN-010) $)_{3}$ AF680-(MC-FN-016) 3 (EDB binder), and control AF680-(MC-FN-0115) $)_{3}$. Mice $(n=3)$ were stratified according to their tumor sizes. $\mathbf{c}$ Kinetics of fluorescence signals in different organs quantified and normalized to the respective organ/tumor weight. Each of the two cystine-knot miniproteins was compared to the control (AF680-(MC-FN-010) $)_{3}$ vs. AF680-(MC-FN-0115) ${ }_{3}$ and AF680-(MC-FN-016) s $_{3}$ vs. AF680-(MC-FN-0115) ${ }_{3}$, respectively) in a given organ at a given time point. d Kinetics of tumor-to-lung, tumor-to-liver, and tumor-to-kidney ratio. Ratios resulting from ex vivo imaging were derived from normalized fluorescence signals after i.v. injection of 3.34 nmol AF680-(MC-FN-010) ${ }_{3}$, AF680-(MC-FN-016) $)_{3}$, and AF680$(\mathrm{MC}-\mathrm{FN}-0115)_{3}$ at 1,2 , and $6 \mathrm{~h}$. e Specific tumor targeting of AF680-(MC-FN-010) ${ }_{3}$ in a competitive experiment. Tumor-to-lung, tumor-to-liver, and tumor-to-kidney ratio of ex vivo imaging are calculated on the basis of the normalized fluorescence signals to the respective tumor weight at 1,2 , and $6 \mathrm{~h}$ (Supplementary Fig. 6). All data are shown as mean \pm SD of triplicates. Statistical significance was calculated with two-way ANOVA $\left({ }^{\star} p<0.05 ;{ }^{\star \star} p<0.01 ;\right.$ $\left.{ }^{\star \star \star} p<0.001\right)$. Source data are provided as a Source Data file.

Table 2 Biodistribution of cystine-knot miniproteins in human U-87 MG xenograft mouse tumors.

\begin{tabular}{|c|c|c|c|c|}
\hline Cystine-knot miniprotein & Tumor-to-organ ratio & $1 \mathrm{~h}$ & $2 \mathrm{~h}$ & $6 \mathrm{~h}$ \\
\hline AF680-(MC-FN-010) 3 & Tumor-to-heart & $8.57 \pm 2.35$ & $18.09 \pm 10.32$ & $14.77 \pm 2.20$ \\
\hline AF680-(MC-FN-010) 3 & Tumor-to-liver & $0.55 \pm 0.06$ & $1.30 \pm 0.40$ & $1.71 \pm 0.71$ \\
\hline AF680-(MC-FN-010) 3 & Tumor-to-kidney & $1.06 \pm 0.17$ & $4.97 \pm 2.89$ & $4.60 \pm 0.19$ \\
\hline AF680-(MC-FN-016) 3 & Tumor-to-heart & $12.86 \pm 6.70$ & $13.58 \pm 10.53$ & $9.88 \pm 3.02$ \\
\hline AF680-(MC-FN-016) 3 & Tumor-to-lung & $1.62 \pm 0.62$ & $2.89 \pm 1.89$ & $3.29 \pm 0.91$ \\
\hline AF680-(MC-FN-016) 3 & Tumor-to-kidney & $0.54 \pm 0.34$ & $1.25 \pm 1.41$ & $1.27 \pm 0.39$ \\
\hline AF680-(MC-FN-0115) 3 & Tumor-to-heart & $3.19 \pm 0.51$ & $4.39 \pm 1.57$ & $3.11 \pm 0.69$ \\
\hline AF680-(MC-FN-0115) 3 & Tumor-to-lung & $0.70 \pm 0.16$ & $1.61 \pm 0.19$ & $1.40 \pm 0.21$ \\
\hline AF680-(MC-FN-0115) $)_{3}$ & Tumor-to-liver & $0.54 \pm 0.13$ & $0.99 \pm 0.16$ & $1.00 \pm 0.23$ \\
\hline AF680-(MC-FN-0115) 3 & Tumor-to-spleen & $1.96 \pm 0.32$ & $2.57 \pm 0.62$ & $1.91 \pm 0.41$ \\
\hline AF680-(MC-FN-0115) 3 & Tumor-to-kidney & $0.09 \pm 0.02$ & $0.20 \pm 0.11$ & $0.21 \pm 0.03$ \\
\hline
\end{tabular}

Tumor-to-organ ratios of $\mathrm{AF680}-(\mathrm{MC}-\mathrm{FN}-010)_{3}, \mathrm{AF680}-(\mathrm{MC}-\mathrm{FN}-016)_{3}$, and AF680-(MC-FN-0115) 3 were calculated based on the fluorescence signals (normalized to the weight) after i.v. injection of $3.34 \mathrm{nmol}$ samples at 1,2 , and $6 \mathrm{~h}(n=3)$. Data are shown as mean \pm SD

The measured binding affinities of the parental MC-FN-010 and its derivative MC-FN-016 to FN-67B89 were in the singledigit micromolar range. As a higher binding strength is required for efficient tumor uptake and retention in vivo, in particular for small peptides that are excreted rapidly via the kidneys ${ }^{39,40}$, we oligomerized these ligands and conjugated the resulting trimers with a fluorescent dye. This resulted in apparent affinities in the picomolar range $(\sim 1500$ - to 3600 -fold stronger as compared to the monomeric versions), comparable with the reported binding constants for the single-chain Fv L19 fragment $^{9}$.

The ability of the trimerized and labeled formats of MC-FN010 and MC-FN-016 to bind to EDB-expressing tumors in vivo was investigated in the U-87 MG xenograft mouse model. Both molecules were selectively taken up and maintained within the tumor over the entire recorded period of $6 \mathrm{~h}$ in contrast to a triple alanine mutant that served as a negative control. Signals observed in liver and kidney decreased over time. The uptake and retention of oMCoTI-II-based peptides in the kidneys was reported in other studies and attributed to a high content of arginine residues ${ }^{41}$. Benchmarking with previously described peptide-based binders, aptide ${ }^{17}$ and nonapeptide ${ }^{19}$, supported the favorable performance of the presented cystine-knot derived probes.

Taken together, the strong tumor uptake of these trimeric constructs and their favorable biodistribution indicate the suitability for diagnostic imaging applications. These EDB ligands may provide a platform, which can support a diversity of in vivo cancer imaging and targeting approaches including conjugation of radioactive tracers, use for PET or SPECT nuclear imaging techniques, and targeting of bioactive payloads.

\section{Methods}

Target expression, purification, and biotinylation. Recombinant human fibronectin EDB, either as a single domain (FN-B, Uniprot ID P02751, isoform 7, amino acid E1265-T1355) or flanked by its surrounding type III domains (FN-67B89, amino acid G1080-E1455), served as target protein in this study while a protein comprising domains 6-9 without EDB (FN-6789) was used as a control. All variants were expressed in E. coli with a c-terminal hexahistidine (H6) tag and purified via IMAC and SEC. For this, codon-optimized DNA sequences were synthesized by Thermo Fisher Scientific, cloned into the pET-21a expression vector (Novagen), and introduced into E. coli BL21 (DE3) cells (Agilent). Proteins were produced at volumes of $750 \mathrm{~mL}$ at $30^{\circ} \mathrm{C}, 120 \mathrm{rpm}$ until an $\mathrm{OD}_{600}$ of approximately 0.7 was reached. For induction of production, $750 \mu \mathrm{L} 1 \mathrm{M}$ IPTG were added to the main culture and incubated at $25^{\circ} \mathrm{C}, 120 \mathrm{rpm}$. overnight. Cells were harvested, resuspended in $10 \mathrm{~mL}$ equilibration buffer $(20 \mathrm{mM}$ Tris- $\mathrm{HCl} \mathrm{pH} 8.0,10 \%$ glycerol, $500 \mathrm{mM} \mathrm{NaCl}, 10 \mathrm{mM}$ imidazole), and lysed by sonification (Branson Digital Sonifier 250). The supernatant was purified by IMAC with a $1 \mathrm{~mL}$ HisTrap column (GE Healthcare) using an ÄKTAprime ${ }^{\text {mit }}$ plus system (GE Healthcare) and a linear gradient from $10-500 \mathrm{mM}$ imidazole in $20 \mathrm{~min}$. Subsequently, target protein containing fractions were combined, dialyzed against phosphate-buffered saline (PBS) $\left(14 \mathrm{mM} \mathrm{NaCl}, 2.7 \mathrm{mM} \mathrm{KCl}, 10 \mathrm{mM} \mathrm{Na}_{2} \mathrm{HPO}_{4}, 1.8 \mathrm{mM} \mathrm{KH}_{2} \mathrm{PO}_{4}, \mathrm{pH} 7.5\right)$ at $4{ }^{\circ} \mathrm{C}$ overnight, and further purified by size exclusion chromatography using a HiLoad 26/600 Superdex $200 \mathrm{pg}$ (for FN-67B89 and FN-6789) or 75 pg (for FN-B) column (GE Healthcare). Final purified proteins were analyzed via SDS-PAGE, analytical SEC, and via ELISA using the EDB-specific BC-1 antibody (ab154210, Abcam). Protein batch purity was furthermore determined via densitometric analysis using ImageQuant software from unmodified SDS-PAGE image. Proteins 
were stored in aliquots in PBS supplemented with 5\% mannitol and 5\% trehalose at $-20^{\circ} \mathrm{C}$. FN-B, FN-67B89 and FN-6789 proteins were biotinylated via primary $\mathrm{NH}_{2}$-groups using EZ-Link Sulfo-NHS-LC-Biotin (Thermo Fisher Scientific) according to the manufacturer's introductions.

Quality control of FN-67B89 target protein. In all, $10 \mu \mathrm{g} / \mathrm{mL}$ target proteins were coated on a Maxisorp ${ }^{\mathrm{TM}}$ 96-well plate in a volume of $100 \mu \mathrm{L}$ via passive absorption under alkaline conditions, using $50 \mathrm{mM} \mathrm{Na}_{2} \mathrm{CO}_{3} \mathrm{pH} 9.4$ coating buffer. Coating of ELISA plates was performed overnight at $4{ }^{\circ} \mathrm{C}$. After removal of coating buffer, the plates were washed three times with $300 \mu \mathrm{L}$ PBS-T and $300 \mu \mathrm{L}$ blocking buffer $(3 \%$ BSA in PBS) was added. Blocking was performed for $2 \mathrm{~h}$ at RT. For primary incubation, plates were washed three times with $300 \mu \mathrm{L}$ PBS-T and incubated for $1 \mathrm{~h}$ with $100 \mu \mathrm{L}$ of BC-1 antibody diluted 1:1000 in PBS-T, at $4{ }^{\circ} \mathrm{C}$. An HRPconjugated anti-mouse antibody (554002, BD Pharmingen ${ }^{\text {"II) }}$ ) diluted 1:5000 in PBS was added after washing three times with PBS-T in $100 \mu \mathrm{L} /$ well and incubated for $1 \mathrm{~h}$ at $4{ }^{\circ} \mathrm{C}$. Plates were then washed three times with PBS-T and three times with PBS prior to detection of antigen-antibody complexes via HRP-mediated conversion of TMB substrate. For this, $100 \mu \mathrm{L}$ TMB substrate was added to the wells and a blue color developed in proportion to the amount of analyte present in the sample. Color development was stopped by adding of $50 \mu \mathrm{L} 0.2 \mathrm{M} \mathrm{HCl}$ per well and the specific absorbance was measured at $450 \mathrm{~nm}$ using an Infinite M200 PRO Microplate Reader (Tecan).

Selection of EDB-specific ligands via phage display. Two different combinatorial libraries, which are based on oMCoTI-II were constructed using an M13 phagemid system. While in the MCopt 1.0 library loop 1 (including a length variation of 6,9 , and 12 amino acids) and loop 3 as well as the two amino acids at the N-terminus have been randomized, the MCopt 2.0 library was built via randomization of loop 1 with 10 amino acids only. Additionally, they are also different in the type of display as MCopt 1.0 is presented via the major coat protein pVIII and MCopt 2.0 via pIII. In total, three screening rounds based on streptavidincoated (SA) magnetic beads were performed with each library. For each screening round $2 \times 50 \mu \mathrm{L}$ Dynabeads ${ }^{\oplus} \mathrm{M}-280$ Streptavidin (Life Technologies) were transferred to a $2 \mathrm{~mL}$ tube each and washed with $1 \mathrm{~mL}$ TBS-T buffer $(50 \mathrm{mM}$ Tris, $150 \mathrm{mM} \mathrm{NaCl}, 0.1 \%$ Tween-20, pH 7.4). $100 \mu \mathrm{g}$ biotinylated FN-B in $200 \mu \mathrm{L}$ TBS ( $50 \mathrm{mM}$ Tris, $150 \mathrm{mM} \mathrm{NaCl}, \mathrm{pH} 7.4$ ) was added to the first tube, $200 \mu \mathrm{L}$ TBS without target protein to the second tube (used for negative selection of phages) and beads were incubated on a rolling mixer for $20 \mathrm{~min}$ at $30 \mathrm{rpm}$. Tubes were placed back into the magnet, remaining solution was discarded, and the beads were washed twice with TBS-T. Beads were blocked with $2 \%$ milk powder (Carl Roth) in TBS for $1 \mathrm{~h}$ at $4{ }^{\circ} \mathrm{C}$ and $30 \mathrm{rpm}$. While biotinylated FN-B-coated SA beads were blocked for another $30 \mathrm{~min}, 7 \times 10^{13}$ (first round) or $7 \times 10^{12}$ (second and third round) phages were added to the uncoated SA beads in $1 \mathrm{~mL} 2 \%$ milk powder in TBS and incubated for $30 \mathrm{~min}$ at RT, $30 \mathrm{rpm}$ (negative selection). The blocking solution of target-coated beads was discarded; beads were washed twice with TBS-T and phage supernatant from negative selection was added. Target-coated beads were incubated with the phage suspension for $1 \mathrm{~h}$ at RT and $30 \mathrm{rpm}$. Subsequently, unbound phages were washed off, washing the beads six times with TBS-T and twice with TBS. To elute bound phages, a pH-shift elution was performed, adding $50 \mu \mathrm{L} 100 \mathrm{mM}$ trimethylamine (TEA) to the washed beads. The TEA bead suspension was incubated for 6 min at max rpm, placed back into the magnet, and supernatant transferred to a fresh tube containing $100 \mu \mathrm{L} 1 \mathrm{M}$ Tris/HCl pH 7 for neutralization. A second elution step was performed by adding $50 \mu \mathrm{L} 100 \mathrm{mM}$ glycine $(\mathrm{pH} 2)$ to the target-coated beads and incubating the mixture for $10 \mathrm{~min}$ in a thermomixer at max rpm. Tubes were placed back into the magnet and the supernatant was transferred to the Tris/TEA mixture from the first elution. Phage eluate was used to infect exponentially growing $E$. coli XL1-Blue (Agilent) for phage pool amplification. In total, $1800 \mu \mathrm{L}$ E. coli XL1-Blue cells at an $\mathrm{OD}_{600}$ of 0.5 were added to the eluate and incubated for $30 \mathrm{~min}$ at $37^{\circ} \mathrm{C}$ without and for $30 \mathrm{~min}$ at $37^{\circ} \mathrm{C}$ with agitation at $150 \mathrm{rpm}$. The infected XL1-Blue cells were then dispended on two large agar plates containing $100 \mu \mathrm{g} / \mathrm{mL}$ carbenicillin and $0.4 \%$ glucose and incubated overnight at $37^{\circ} \mathrm{C}$, before phage rescue was performed on the following day. For phage rescue, $4 \mathrm{~mL}$ LB medium was distributed per plate and cells scraped off with a cell scraper. A $50-\mathrm{mL}$ culture was inoculated to a final $\mathrm{OD}_{600}$ of 0.2 . Cells were grown until an $\mathrm{OD}_{600}$ of 0.5 and infected with $0.5 \times 10^{12}$ VCSM13 helper phages (Agilent) for phage production from phagemid vector. E. coli XL1-Blue cells were incubated for $30 \mathrm{~min}, 37^{\circ} \mathrm{C}$, without agitation followed by $30 \mathrm{~min}, 37^{\circ} \mathrm{C}$, with agitation at $150 \mathrm{rpm}$. The bacterial suspension was centrifuged at $4500 \times \mathrm{g}$ for $10 \mathrm{~min}$ at RT and the supernatant was discarded. Bacterial pellets were taken up in $50 \mathrm{~mL}$ LB medium (supplemented with $100 \mu \mathrm{g} / \mathrm{mL}$ carbenicillin, $25 \mu \mathrm{g} / \mathrm{mL}$ kanamycin, $1 \mathrm{mM}$ IPTG) for induction of protein production and E. coli phage production cultures grown overnight at $30^{\circ} \mathrm{C}, 250 \mathrm{rpm}$. E. coli cells were harvested by centrifugation at $4500 \times g$ for $15 \mathrm{~min}, 4^{\circ} \mathrm{C} .10 \mathrm{~mL}$ PEG/NaCl solution $(25 \%(\mathrm{w} / \mathrm{v})$ polyethylene glycol, $15 \%(\mathrm{w} / \mathrm{v}) \mathrm{NaCl}$ ) were added to $40 \mathrm{~mL}$ of the phage containing supernatant, inverted, and placed on ice for $30 \mathrm{~min}$. Phage particles were precipitated via centrifugation at $15,000 \times g, 20 \mathrm{~min}$ at $4{ }^{\circ} \mathrm{C}$. Supernatants were discarded and phage pellets were taken up in $1600 \mu \mathrm{L}$ Tris/HCl ( $\mathrm{pH}$ 8.0) for subsequent centrifugation at $15,000 \times g, 10 \mathrm{~min}$ at $4{ }^{\circ} \mathrm{C}$. The phage containing supernatant was added to $400 \mu \mathrm{L} \mathrm{PEG} / \mathrm{NaCl}$ again and incubated on ice for $20 \mathrm{~min}$. After the second $\mathrm{PEG} / \mathrm{NaCl}$ precipitation, tubes were centrifuged at $15,000 \times g$,
$15 \mathrm{~min}$ at $4{ }^{\circ} \mathrm{C}$. Phage pellets were re-suspended in $800 \mu \mathrm{L}$ Tris/ $\mathrm{HCl}$ and heated to $65^{\circ} \mathrm{C}$ for $15 \mathrm{~min}$ in a final purification step. Phage suspensions were finally centrifuged at RT, $15,000 \times g$ for $10 \mathrm{~min}$, supernatants were taken and phage particle concentration was determined photometrically using the dual wavelength modus $\mathrm{OD}_{269}-\mathrm{OD}_{320}$. Phage concentrations were calculated according to the nucleotide content and molar extinction coefficient of M13 phages ${ }^{42}$.

Hit identification of selected cystine-knot miniproteins. In the identification process, enriched screening pool phagemid vectors derived from the third screening round were prepared in order to amplify cystine-knot miniprotein sequences by PCR. PCR-inserts were cloned into the expression vector pET-32LibEx, a derivative of pET-32a (Novagen), to enable an expression of cystine-knot miniproteins as Thioredoxin-A fusion variants. This vector carries DNA sequences in successive order encoding for E. coli Thioredoxin-A to allow efficient disulfide bond formation in the cytoplasm in combination with an oxidoreductase knockout strain ${ }^{43}$, an H6-tag for rapid purification, an S-tag for detection by an antibody, and a thrombin cleavage site to remove the fusion tag. DNA fragments encoding for cystine-knot miniprotein sequences were introduced downstream of the thrombin cleavage site into pET-32-LibEx vector via unique BamHI and KpnI restriction sites. Vectors were introduced into E. coli SHuffle ${ }^{\circledast}$ T7 Express cells (New England BioLabs) via heat shock and the cells were plated on selective agar plates. Single colonies were picked, sequenced, and transferred to a 96-well plate for small-scale expression in $1 \mathrm{~mL}$ autoinduction medium (MagicMedia" ${ }^{\text {"w }}$; Thermo Fisher Scientific). Protein production was conducted overnight at $30^{\circ} \mathrm{C}$ and $220 \mathrm{rpm}$. Cells were harvested by centrifugation at $3000 \times g$ for $15 \mathrm{~min}$, lysed via incubation in buffer $(20 \mathrm{mM}$ Tris, $2 \mathrm{mM} \mathrm{MgCl}, 20 \mathrm{mM} \mathrm{NaCl}, \mathrm{pH}$ 8) containing $0.1 \mathrm{mg} / \mathrm{mL}$ lysozyme (Merck Millipore) and $5 \mathrm{U} / \mathrm{mL}$ benzonase (Merck Millipore) combined with a freeze-thaw cycle, and heated for $10 \mathrm{~min}$ at $80^{\circ} \mathrm{C}$. After a final centrifugation $\left(3000 \times g, 15 \mathrm{~min}, 4^{\circ} \mathrm{C}\right)$ to remove cell debris, the supernatant was collected for E-PAGE ${ }^{\mathrm{m}}$ and binding analysis. E-PAGE ${ }^{\mathrm{TM}}$ (Life Technologies) was used as a high throughput gel system to simultaneously analyze 96-probes in parallel for a quantification of produced and heat-purified proteins. E-PAGE ${ }^{\mathrm{TM}}$ analysis was performed according to the manufacturer's instructions. ELISA was used as a technique to analyze protein-protein interactions. Cavities of 96-well microtiter plates (Nunc MaxiSorp ${ }^{\mathrm{m}}$; Thermo Fisher Scientific) were coated with $1 \mu \mathrm{g}$ FN-B, BSA (Eurobio), milk powder, streptavidin (Sigma Aldrich), T7-HisTEV-B (LD BioPharma), FN-B(8-14) (R\&D Systems), lysozyme, ovalbumin (GE Healthcare Life Science), aldolase (GE Healthcare Life Science), or $0.6 \mu \mathrm{g}$ anti-cmyc antibody (M4439; Sigma) overnight at $4{ }^{\circ} \mathrm{C}$. The wells were washed three times with PBS-T ( $1 \times$ PBS with $0.1 \%(w / v)$ Tween-20), blocked with $1 \times$ Casein buffer (Sigma Aldrich) diluted in PBS for $2 \mathrm{~h}$ at RT, and washed again as described. $20 \mu \mathrm{L}$ of supernatant containing the respective heat-purified fusion protein were added to $80 \mu \mathrm{L}$ PBS-T or $200 \mathrm{nM}$ of MC-Myc-010 fusion protein (wells with anti-cmyc antibody) diluted in PBS-T, applied to the cavities, and incubated for $1 \mathrm{~h}$ at $4^{\circ} \mathrm{C}$. After three times of washing with PBS-T, binding of the respective variant was detected with a horseradish peroxidase (HRP)-conjugated anti-S-tag antibody (ab18589 or ab19324; Abcam) 1:2000 diluted in PBS. Enzymatic reaction was measured with TMB as a chromogenic substrate and stopped with $0.2 \mathrm{M} \mathrm{HCl}$ after approximately $5 \mathrm{~min}$. The measurement of absorbance at $450 \mathrm{~nm}$ was performed using an Infinite M200 PRO Microplate Reader (Tecan). In order to compare binding signals among different plates, ELISA signals were normalized to the internal plate control (c-myc antibody binding). Normalized FN-B signals were then referenced to normalized BSA signals to evaluate binding ability of selected cystine-knot miniproteins. In addition, the target-binding signals were correlated to the protein expression rate, resulting in a ranking value for the identification of hits.

Recombinant cystine-knot miniprotein production. Recombinant protein production was carried out using E. coli SHuffle ${ }^{\varpi}$ T7 Express cells carrying pET-32LibEx vector encoding for the respective cystine-knot miniprotein sequence in a $750-\mathrm{mL}$ scale at $30^{\circ} \mathrm{C}, 120 \mathrm{rpm}$. After the culture reached an $\mathrm{OD}_{600}$ of approximately 0.7 , induction of production was achieved by adding $750 \mu \mathrm{L} 1 \mathrm{M}$ IPTG and incubation at $25^{\circ} \mathrm{C}, 120 \mathrm{rpm}$ overnight. E. coli cells were harvested, re-suspended in $10 \mathrm{~mL}$ equilibration buffer, lysed by sonification, and heated to $80^{\circ} \mathrm{C}$ for $10 \mathrm{~min}$. After centrifugation of cell debris $\left(15,000 \times g\right.$ for $\left.30 \mathrm{~min}, 4^{\circ} \mathrm{C}\right)$, the supernatant was purified by IMAC with a $1 \mathrm{~mL}$ HisTrap column using an ÄKTAprime ${ }^{\mathrm{m}}$ plus system and a linear gradient from 10 to $500 \mathrm{mM}$ imidazole in $20 \mathrm{~min}$. The cystine-knot miniprotein fusion protein (Trx-cystine-knot miniprotein) containing fractions were collected and dialyzed against thrombin cleavage buffer $(20 \mathrm{mM}$ Tris, $150 \mathrm{mM}$ $\mathrm{NaCl}, 1.5 \mathrm{mM} \mathrm{CaCl}_{2}$, and $5 \%\left(\mathrm{w} / \mathrm{v}\right.$ ) glycerol, $\mathrm{pH} 8.45$ ) at $4{ }^{\circ} \mathrm{C}$ overnight. Trxcystine-knot miniproteins were either directly used for ELISA-based assays or processed further in case that the untagged miniprotein was needed, e.g. for SPR analysis. For this, Trx-cystine-knot miniproteins were cleaved with $0.5 \mathrm{U}$ of thrombin (Sigma Aldrich) per $1 \mathrm{mg}$ protein and incubated at $37^{\circ} \mathrm{C}$ overnight. Separation of cleaved protein fragments was performed by reverse phase chromatography with an Agilent 1260 Infinity Quaternary LC system (Agilent) and a $3 \mathrm{~mL}$ RESOURCE ${ }^{\mathrm{m}} \mathrm{RPC}$ column (GE Healthcare) using a linear gradient from $2 \%$ to $80 \%$ acetonitrile in $\mathrm{H}_{2} \mathrm{O}$ supplemented with $0.05 \%$ trifluoroacetic acid (TFA). Respective fractions containing cystine-knot miniprotein were lyophilized in a RVC 2-18-CD Plus rotational vacuum concentrator (Christ) as a final step. 
Amount of cystine-knot miniprotein was determined by weighing and the peptides were stored in lyophilized form at $-20^{\circ} \mathrm{C}$. Identity was verified by mass spectrometry with a LCMS Single Quad G6130B System (Agilent Technologies) using a standard electrospray ionization protocol.

Alanine scanning mutagenesis of selected MC-FN-010. For generation of alanine scanning MC-FN-010 derivatives mutations were either introduced via PCR or the whole coding sequence was assembled via direct synthesis of GeneArt ${ }^{\text {tw }}$ Strings ${ }^{\text {tw1 }}$ fragments (Thermo Fisher Scientific). Respective DNA fragments were cloned into pET-32-LibEx expression vector using unique BamHI and KpnI restriction sites and introduced into E. coli SHuffle ${ }^{\circledR}$ T7 Express competent cells (New England BioLabs). All mutations were verified by DNA sequencing. The alanine scanning mutagenesis variants were expressed in 24-well format using $5 \mathrm{~mL}$ of selective autoinduction medium. Production and Trx-cystine-knot miniprotein purification were performed as described above for the 96-well format, but included a further purification step of the supernatant using HisPur ${ }^{\mathrm{m}} \mathrm{Ni}$-NTA spin columns (Thermo Fisher Scientific). Binding ability and specificity of Trx-cystineknot miniproteins to target and off-target protein was carried out with an antibody-based ELISA assay as described above.

Surface plasmon resonance spectroscopy. Binding kinetics of monomeric and trimeric cystine-knot miniprotein ligands to its target protein was determined using a Biacore T-100 device (GE Healthcare Life Science) with PBS-T as running buffer. For this, the biotinylated FN-67B89 protein $(200-300 \mu \mathrm{g} / \mathrm{mL})$ was captured by binding to a flow cell of an SA sensor chip (GE Healthcare Life Science). To analyze monomeric ligands, an immobilized target density of maximum 750 response units (RU) was applied and for trimeric variants a RU of maximum 400 was aimed for. Binding analysis of monomeric ligands was performed using a multi-cycle kinetic method with concentrations ranging from 50 to $4000 \mathrm{nM}$. A cycle started with an association period of $90 \mathrm{~s}$, followed by a dissociation period of $420 \mathrm{~s}$ and a final regeneration step. Kinetic measurement was conducted applying a flow rate of $20 \mu \mathrm{L} / \mathrm{min}$. Trimeric variants were analyzed under the same association and dissociation conditions, but using the single-cycle kinetic measurement mode in a constant flow of $30 \mu \mathrm{L} / \mathrm{mL}$. In this case the analyte concentration was between 1.25 and $10 \mathrm{nM}$. Binding kinetics and steady-state analysis were calculated using a global kinetic fit model (1:1 Langmuir, Biacore T-100 Evaluation Software; GE Healthcare Life Science)

Immunofluorescence staining. Cryopreserved tumor pieces were cut in $6-\mu \mathrm{m}-$ thick sections, fixed in ice cold acetone for $5 \mathrm{~min}$, and air-dried. Slides were then blocked in PBS with 3\% BSA at RT for $5 \mathrm{~min}$. For staining of EDB, $1 \mu \mathrm{g}$ of the respective biotinylated cystine-knot miniprotein was incubated with $2.9 \mu \mathrm{g}$ streptavidin-Cy3 conjugate (Rockland Immunochemicals) at RT for $30 \mathrm{~min}$. The pre-formed complex or $0.1 \mu \mathrm{g}$ AF680-(MC-FN-010) ${ }_{3}$ and anti-mouse CD31 antibody (RB-10333-P1, Thermo Fisher) diluted 1:100 in PBS with 1\% BSA was then added to the tumor sections and incubated for $30 \mathrm{~min}$ at $37^{\circ} \mathrm{C}$. Afterwards, slides were washed three times with PBS containing $1 \%$ BSA. CD31 staining of sections treated with pre-formed complex was performed with a rat anti-mouse CD31 IgG antibody (102416; BioLegend) diluted 1:100 in PBS with 1\% BSA for $30 \mathrm{~min}$ at $37^{\circ} \mathrm{C}$ and detected with a secondary anti-rabbit IgG-Cy3 antibody (111-165-003; Jackson ImmunoResearch) diluted 1:400 in PBS with $1 \%$ BSA for 30 min at $37^{\circ} \mathrm{C}$. After three washing steps in PBS, cell nuclei were stained with Höchst 33342 (Thermo Fisher Scientific) diluted 1:5000 in PBS for $30 \mathrm{~min}$ at RT. Slides were washed again as described above and covered with coverslips in a thin layer of mounting medium (Dako). Images were captured with a Zeiss Apotome microscope (Carl Zeiss) and analyzed with ZEN software (Carl Zeiss).

Peptide synthesis. Trimeric ligands as well as N-terminally biotinylated miniproteins were purchased from Pepscan. Cystine-knot miniproteins were generated via conventional solid phase peptide synthesis (SPPS). The oligomerization strategy of trimeric ligands was similar to the described procedure of Kim et al. ${ }^{44}$, but using a triple GSGSK-S spacer peptide that served as a trimerization handle. AF680labeled aptide and nonapeptide was synthesized according to the same protocol of Kim et al. ${ }^{17}$ or Han et al. ${ }^{19}$, respectively. All obtained peptidic constructs are listed in Supplementary Table 3 and stored as $100 \mu \mathrm{g}$ aliquots at $-20^{\circ} \mathrm{C}$. For experiments all peptides were dissolved in $100 \mu \mathrm{L}$ DPBS (Gibco) resulting in a concentration of $1 \mu \mathrm{g} / \mu \mathrm{L}$. For all constructs identity was verified by ESI mass spectrometry and purity was analyzed by analytical reverse phase chromatography (performed by Pepscan, see Supplementary Figs. 9 and 10 and Supplementary Tables 3 and 4). Additionally, trimers were analyzed via SDS-PAGE and SPR in order to characterize target-binding properties (binding to FN-67B89, see Supplementary Fig. 3b) and specificity (binding to FN-6789).

U-87 MG xenograft mouse model. Human glioblastoma U-87 MG (ATCC) cell line was cultured in EMEM medium (ATCC) supplemented with 10\% FCS under aseptic conditions at $37^{\circ} \mathrm{C}$ with $5 \% \mathrm{CO}_{2}$ and $95 \%$ humidity. Cell lines from ATCC have been thoroughly analyzed and authenticated to ensure their identity and quality. Additionally, we verified the cell line via morphology examination, NGS sequencing, and STR profiling and tested them negatively for mycoplasma contamination. All mice were kept in accordance with federal and state policies on animal research at BioNTech SE. Procedures and experimental group sizes were approved by the regulatory authorities (Tierschutzkommision des Landesuntersuchungsamts Rheinland-Pfalz) for animal welfare. Four-week-old Fox n1/ nu mice ranging in weights between approximately 25 and $28 \mathrm{~g}$ were obtained from Janvier Labs. For xenograft mouse studies $7 \times 10^{6}$ human U-87 MG cells were subcutaneously injected into the right flank of Fox n1/nu mice and tumors were allowed to grow for approximately 5 weeks. Subcutaneous tumor size was determined using ellipsoid formula $\left(\left(\right.\right.$ width $\times$ length $\left.\left.\left.^{2}\right) / 2\right)\right)$. All animals with tumor volume between 50 and $1500 \mathrm{~mm}^{3}$ were included in the studies and mice were randomly assigned to experimental cohorts.

In vivo and ex vivo imaging. Mice carrying a desired tumor size were included for analysis of biodistribution and tumor targeting of trimeric constructs and peptides. All trimeric constructs and peptides were injected intravenously via retrobulbar venous plexus in a final volume of $100 \mu \mathrm{L}$ PBS buffer $(3.34 \mathrm{nmol}, 10 \mathrm{nmol}$, or $34.7 \mathrm{nmol} / \mathrm{mice}$ ). Mice ( $n=3$ for each construct) were imaged in an IVIS Spectrum System (Perkin Elmer) using excitation range of $615-665 \mathrm{~nm}$ and monitoring emission signals at $695-770 \mathrm{~nm}$. Imaging process was performed 1, 2, 3, or $6 \mathrm{~h}$ post-injection and after euthanization the tumor and specific organs were excised, imaged, weighed, and cryo-conservated for further analysis. Fluorescence intensity of regions of interest was quantified using Living Image ${ }^{\circledR}$ software (Perkin Elmer).

Statistical analysis. Statistical significance was calculated based on triplicate data sets using two-way ANOVA analysis with corrected $p$ values (Sidak's method) for comparison of compounds and between tissue types in GraphPad Prism 6. ${ }^{*} p<0.05$; ${ }^{* *} p<0.01 ;{ }^{* * *} p<0.001$. All data are presented as mean \pm SD.

Reporting summary. Further information on research design is available in the Nature Research Reporting Summary linked to this article.

\section{Data availability}

The source data underlying Figs. 2, 3, 4 and Supplementary Figs. 1, 2, 3, 4, 7 are provided as a Source Data file. Any other data are available from the authors upon reasonable request.

Received: 12 December 2018; Accepted: 28 November 2019; Published online: 15 January 2020

\section{References}

1. Gwyther, S. J. New imaging techniques in cancer management. Ann. Oncol. 16 (Suppl 2), ii63-ii70 (2005)

2. Massoud, T. F. \& Gambhir, S. S. Molecular imaging in living subjects: seeing fundamental biological processes in a new light. Genes Dev. 17, 545-580 (2003).

3. Drevs, J. \& Schneider, V. The use of vascular biomarkers and imaging studies in the early clinical development of anti-tumour agents targeting angiogenesis. J. Intern. Med. 260, 517-529 (2006).

4. Castellani, P. et al. Differentiation between high- and low-grade astrocytoma using a human recombinant antibody to the extra domain-B of fibronectin. Am. J. Pathol. 161, 1695-1700 (2002).

5. Neri, D. \& Bicknell, R. Tumour vascular targeting. Nat. Rev. Cancer $\mathbf{5}$, 436-446 (2005)

6. Schwarzbauerl, J. E. Multiple sites of alternative splicing of the rat fibronectin gene transcript. EMBO J. 6, 2573-2580 (1987).

7. Castellani, P. et al. The fibronectin isoform containing the ed-b oncofetal domain. A marker of angiogenesis. Int. J. Cancer 59, 612-618 (1994).

8. Kumra, H. \& Reinhardt, D. P. Fibronectin-targeted drug delivery in cancer. Adv. Drug Deliv. Rev. 97, 101-110 (2016).

9. Pini, A. et al. Design and use of a phage display library human antibodies with subnanomolar affinity against a marker of angiogenesis eluted from a twodimensional gel. J. Biol. Chem. 273, 21769-21776 (1998).

10. Sauer, S. et al. Expression of the oncofetal ED-B-containing fibronectin isoform in hematologic tumors enables ED-B-targeted 131I-L19SIP radioimmunotherapy in Hodgkin lymphoma patients. Blood 113, 2265-2274 (2009).

11. Pasche, N. \& Neri, D. Immunocytokines: a novel class of potent armed antibodies. Drug Discov. Today 17, 583-590 (2012).

12. Demartis, S., Tarli, L., Borsi, L., Zardi, L. \& Neri, D. Selective targeting of tumour neovasculature by a radiohalogenated human antibody fragment specific for the ED-B domain of fibronectin. Eur. J. Nucl. Med. 28, 534-539 (2001).

13. Fabbrini, M. et al. Selective occlusion of tumor blood vessels by targeted delivery of an antibody-photosensitizer conjugate. Int. J. Cancer 118, 1805-1813 (2006). 
14. Chames, P., van Regenmortel, M., Weiss, E. \& Baty, D. Therapeutic antibodies: successes, limitations and hopes for the future. Br. J. Pharmacol. 157, 220-233 (2009).

15. Lorey, S. et al. Novel ubiquitin-derived high affinity binding proteins with tumor targeting properties. J. Biol. Chem. 289, 8493-8507 (2014).

16. Gebauer, M., Schiefner, A., Matschiner, G. \& Skerra, A. Combinatorial design of an Anticalin directed against the extra-domain $b$ for the specific targeting of oncofetal fibronectin. J. Mol. Biol. 425, 780-802 (2013).

17. Grabulovski, D., Kaspar, M. \& Neri, D. A novel, non-immunogenic Fyn SH3derived binding protein with tumor vascular targeting properties. J. Biol. Chem. 282, 3196-3204 (2007).

18. Han, Z. et al. EDB fibronectin specific peptide for prostate cancer targeting. Bioconjugate Chem. 26, 830-838 (2015).

19. Kim, S. et al. Bio-inspired design and potential biomedical applications of a novel class of high-affinity peptides. Angew. Chem. Int. Ed. Engl. 51, 1890-1894 (2012).

20. Kimura, R. H., Cheng, Z., Gambhir, S. S. \& Cochran, J. R. Engineered knottin peptides: a new class of agents for imaging integrin expression in living subjects. Cancer Res. 69, 2435-2442 (2009).

21. Kittle, D. S. et al. Fluorescence-guided tumor visualization using the tumor paint BLZ-100. Cureus https://doi.org/10.7759/cureus.210 (2014).

22. Zhu, X. et al. $99 \mathrm{mTc}$-labeled cystine knot peptide targeting integrin $\alpha \mathrm{v} \beta 6$ for tumor SPECT imaging. Mol. Pharm. 11, 1208-1217 (2014).

23. Avrutina, O. Synthetic cystine-knot miniproteins-valuable scaffolds for polypeptide engineering. Adv. Exp. Med. Biol. 917, 121-144 (2016).

24. Moore, S. J. et al. Engineered knottin peptide enables noninvasive optical imaging of intracranial medulloblastoma. Proc. Natl Acad. Sci. USA 110, 14598-14603 (2013).

25. Kintzing, J. R. \& Cochran, J. R. Engineered knottin peptides as diagnostics, therapeutics, and drug delivery vehicles. Curr. Opin. Chem. Biol. 34, 143-150 (2016).

26. Avrutina, O. et al. Trypsin inhibition by macrocyclic and open-chain variants of the squash inhibitor MCoTI-II. Biol. Chem. 386, 1301-1306 (2005).

27. Carnemolla, B., Leprini, A., Allemanni, G., Saginati, M. \& Zardi, L. The inclusion of the type III repeat ED-B in the fibronectin molecule generates conformational modifications that unmask a cryptic sequence. J. Biol. Chem. 267, 24689-24692 (1992).

28. Mao, Y. \& Schwarzbauer, J. E. Fibronectin fibrillogenesis, a cell-mediated matrix assembly process. Matrix Biol. 24, 389-399 (2005).

29. Mariani, G. et al. Tumor targeting potential of the monoclonal antibody BC-1 against oncofetal fibronectin in nude mice bearing human tumor implants. Cancer 80, 2378-2384 (1997).

30. Albrecht, V. et al. Anticalins directed against the fibronectin extra domain B as diagnostic tracers for glioblastomas. Int. J. Cancer 138, 1269-1280 (2016)

31. Mohammadgholi, M. et al. Human fibronectin extra-domain B (EDB)specific aptide (APTEDB) radiolabelling with technetium- $99 \mathrm{~m}$ as a potent targeted tumour-imaging agent. Anti-Cancer Agents Med. Chem. 18, 277-285 (2017).

32. Haque, A., Faizi, M. S. H., Rather, J. A. \& Khan, M. S. Next generation NIR fluorophores for tumor imaging and fluorescence-guided surgery: A review. Bioorg. Med. Chem. 25, 2017-2034 (2017).

33. Tijink, B. M. et al. Radioimmunotherapy of head and neck cancer xenografts using 131I-labeled antibody L19-SIP for selective targeting of tumor vasculature. J. Nucl. Med. 47, 1127-1135 (2006).

34. Hemmerle, T. et al. The antibody-based targeted delivery of TNF in combination with doxorubicin eradicates sarcomas in mice and confers protective immunity. Br. J. Cancer 109, 1206-1213 (2013).

35. Johannsen, M. et al. The tumour-targeting human L19-IL2 immunocytokine: preclinical safety studies, phase I clinical trial in patients with solid tumours and expansion into patients with advanced renal cell carcinoma. Eur. J. Cancer 46, 2926-2935 (2010).

36. Danielli, R. et al. Intralesional administration of L19-IL2/L19-TNF in stage III or stage IVM1a melanoma patients: results of a phase II study. Cancer Immunol. Immunother. 64, 999-1009 (2015).

37. Weide, B. et al. Intralesional treatment of stage III metastatic melanoma patients with L19-IL2 results in sustained clinical and systemic immunologic responses. Cancer Immunol. Res. 2, 668-678 (2014).

38. Fattorusso, R. et al. NMR structure of the human oncofoetal fibronectin ED-B domain, a specific marker for angiogenesis. Structure 7, 381-390 (1999).
39. Schmidt, M. M. \& Wittrup, K. D. A modeling analysis of the effects of molecular size and binding affinity on tumor targeting. Mol. Cancer Ther. 8 , 2861-2871 (2009)

40. Zahnd, C. et al. Efficient tumor targeting with high-affinity designed ankyrin repeat proteins: effects of affinity and molecular size. Cancer Res. 70, 1595-1605 (2010).

41. Kimura, R. H. et al. Pharmacokinetically stabilized cystine knot peptides that bind alpha-v-beta- 6 integrin with single-digit nanomolar affinities for detection of pancreatic cancer. Clin. Cancer Res. 18, 839-849 (2012).

42. Barbas, C. F., Burton, D. R., Scott, J. K. \& Silverman, G. J. Phage Display (Cold Spring Harbor Laboratory Pr, 2001).

43. Lobstein, J. et al. SHuffle, a novel Escherichia coli protein expression strain capable of correctly folding disulfide bonded proteins in its cytoplasm. Microb. Cell Fact. 11, 56 (2012).

44. Kim, J. W., Cochran, F. V. \& Cochran, J. R. A chemically cross-linked knottin dimer binds integrins with picomolar affinity and inhibits tumor cell migration and proliferation. J. Am. Chem. Soc. 137, 6-9 (2015).

\section{Acknowledgements}

We thank K. Reupke and M. Fierek for their technical assistance, N. Krimmel, K. Thiemann, K. Walzer, B. Jesionek, N. Brüne, D. Schneider, S. Kühne, M. Pfeifer, S. Wöll, F. Vascotto, and E. Stanganello for technical support and advice and K. Chu for proofreading of the manuscript.

\section{Author contributions}

B.G.L., J.W.-L., M.D., H.-U.S. and U.S. are responsible for conceptual design of the study. B.G.L., N.S., J.W.-L. and H.-U.S. performed the experiments. B.G.L., N.S., J.W.-L., M.D and H.-U.S. analyzed and interpreted the data. B.G.L., H.-U.S. and Ö.T. wrote the manuscript. All authors critically revised the manuscript.

\section{Competing interests}

B.G.L., J.W.-L., M.D., H.-U.S., Ö.T., and U.S. are associated with BioNTech SE (Mainz Germany), a company that develops diagnostic and therapeutic compounds based on peptidic ligands. B.G.L., N.S., J.W.-L., M.D., H.-U.S., and U.S. have filed a patent application that includes the data described in this article. U.S. is CEO, cofounder, and shareholder of BioNTech and has ownership interest (including patents).

\section{Additional information}

Supplementary information is available for this paper at https://doi.org/10.1038/s41467019-13948-y.

Correspondence and requests for materials should be addressed to U.S.

Peer review information Nature Communications thanks the anonymous reviewer(s) for their contribution to the peer review of this work.

Reprints and permission information is available at http://www.nature.com/reprints

Publisher's note Springer Nature remains neutral with regard to jurisdictional claims in published maps and institutional affiliations.

Open Access This article is licensed under a Creative Commons Attribution 4.0 International License, which permits use, sharing, adaptation, distribution and reproduction in any medium or format, as long as you give appropriate credit to the original author(s) and the source, provide a link to the Creative Commons license, and indicate if changes were made. The images or other third party material in this article are included in the article's Creative Commons license, unless indicated otherwise in a credit line to the material. If material is not included in the article's Creative Commons license and your intended use is not permitted by statutory regulation or exceeds the permitted use, you will need to obtain permission directly from the copyright holder. To view a copy of this license, visit http://creativecommons.org/ licenses/by/4.0/

(C) The Author(s) 2020 